\title{
Rethinking Short-Term Electricity Market Design: Options for Market Segment Integration
}

by

\author{
Susanne Rieß ${ }^{1}$, Christoph Neumann ${ }^{1}$, Samuel Gließmann², \\ Michael Schöpf, Gilbert Fridgen
}

in: Proceedings of the 14th International Conference on the European Energy Market (EEM), Dresden, Germany, June 2017, p. 1-6

\footnotetext{
1 TenneT TSO GmbH

2 TenneT TSO B.V.
} 


\section{Rethinking Short-Term Electricity Market Design: Options for Market Segment Integration}

\author{
Susanne Rieß \\ Christoph Neumann \\ TenneT TSO GmbH \\ Bayreuth, Germany \\ christoph.neumann@tennet.eu
}

\author{
Samuel Glismann \\ TenneT TSO B.V. \\ Arnhem, The Netherlands
}

\author{
Michael Schoepf \\ Gilbert Fridgen \\ University of Bayreuth \\ Bayreuth, Germany
}

\begin{abstract}
Electricity market design varies across countries throughout Europe. Thereby the provision and remuneration of flexibility always takes place in short-term market segments. Taking into consideration the fundamental changes of the power system, this paper discusses options for the future short-term market design. We develop a conceptual basis for a possible integration of currently separated short-term market segments. Market segment integration (MSI) is defined as the interaction between and possible combination of market segments, i.e. intraday market (ID), congestion management (CM) and balancing market (BA). The paper especially focusses on two options, namely an integrated $\mathrm{BA}$ and $\mathrm{CM}$ market and an integrated ID and CM market. For these options we determine the basic design features. We propose a criteria catalogue which allows the evaluation of the market design options. Based on several criteria we discuss possible positive and negative consequences as well as potential solutions.
\end{abstract}

Index Terms - balancing market; congestion management; intraday market; market design; market segment integration

\section{INTRODUCTION}

Driven by EU regulation, Transmission System Operators (TSOs) and Power Exchanges are working on market integration by coupling (national) short-term power markets to reach the EU target model. In addition to these well-known activities, we go a step further and develop a conceptual basis for a possible integration of separated market segments. The (market) segments referred to are the intraday market (ID), the balancing market (BA), and congestion management ${ }^{1}(\mathrm{CM})$. We thereby question the current structure of short-term market segments as the same flexibility can be offered and used for multiple purposes. Flexibility providers need to choose where to offer their services, being affected in their decision due to overlapping trading times, different market prices, and the variety of product specifications. An option to prevent possible resulting problems like a liquidity split is the

\footnotetext{
${ }^{1}$ Certainly, the term $\mathrm{CM}$ includes a variety of measures, whereas we focus on congestion problems within a bidding zone (see also II A.) Although there is no market-based approach for redispatch procedures implemented in Germany, we use the term "market segment" for pragmatic reasons for all three discussed segments.
}

integration of these fragmented short-term markets. By market segment integration (MSI) we understand the interaction between and possible combination of market segments, i.e. ID, $\mathrm{CM}$ and BA. A co-optimization, for instance, of the BA and $\mathrm{CM}$ processes could not only unlock flexibility to the system but also holds a potential for increasing the overall economic benefit. Moreover current EU legislation [1] suggests the implementation of a market-based approach for dealing with congestions what gives another impetus to rethink the short-term electricity market design.

\section{STRUCTURE AND PRINCIPLES OF SHORT-TERM ELECTRICITY MARKETS}

\section{A. Description of the Status-Quo}

In the current short-term market design of Germany, the market segments ID, BA and CM are strictly separated. The rationales and structure of each market segment will be discussed in the following, focusing on the German case.

Although electricity spot markets consist of the day-ahead market and the ID we will focus on the latter one as flexibility is being traded here in the short-term starting after 3 p.m. on the day before delivery until gate closure time (GCT) 30 minutes before delivery. Market participants can optimize their portfolio in order to uphold balancing group commitments with regards to the imbalance settlement period (ISP) of 15 minutes. As the current market design is based on a zonal approach, the location of the bid is irrelevant. The ID is a two-sided trading that takes place over-the-counter or at power exchanges - in the case of Germany at EPEX SPOT and Nord Pool. Market parties can trade quarter hourly products in the ID auction and/or quarter-hourly respectively hourly products in the continuous intraday trading. Especially due to the increased penetration of wind and solar energy, the need to update production forecasts led to a strong increase of trading volume in the intraday time frame. The traded volume at the power exchange EPEX SPOT increased from 6 TWh in 2009 to 41 TWh in 2016 in the German-Austrian bidding zone, [2], [3].

The BA is designed with a single-sided buyer auction with the TSO as single buyer that has to ensure the balance 
between electricity generation and consumption within the control area at all times. On the BA the products Frequency Containment Reserves (FCR), Automatic Frequency Restoration Reserves (aFRR) and Manual Frequency Restoration Reserves (mFRR) are tendered. There are different products for positive and negative capacity in aFRR and mFRR. FCR is tendered as a symmetric product. The TSO reserves capacities for all three products. The remuneration of the products is split into the capacity price (€/MW), which is paid for every accepted bid, and - in case of aFRR and mFRR - the energy price ( $€ / M W h)$, which is paid for every activated bid. The acceptance of bids is determined by the merit order of the capacity price. The order of activation is determined by the merit order of the energy prices, whereas the pricing method (at least for the time being) is pay-as-bid. Also, for this market segment, the location of participating units is irrelevant and furthermore, a pooling on control area level is allowed [4]. On average, in the year 2016 capacities of around $2000 \mathrm{MW}$ were reserved for positive and negative mFRR. However, mFRR is used in times of high imbalances only. In 2016, around 0.174 TWh (positive balancing energy) and $0.054 \mathrm{TWh}$ (negative balancing energy) was used for balancing the system.

As future development, there is a discussion on the introduction of a balancing energy market for mFRR and potentially aFRR close to delivery as a complement to the existing scheme [5]. Market participants with remaining flexibility could bid an energy price ( $€ / \mathrm{MWh})$ into the balancing energy market without participating in the capacity auction beforehand. This creates an increased competition for balancing energy prices and extends the balancing energy merit-order list. The TSO then is using the entire merit-order list for the respective activation, without distinction between procured and non-procured bids.

As a third segment, $\mathrm{CM}$ is necessary if grid capacity is scarce and measures are needed to prevent or resolve poweror voltage-related violations in the grid. Congestions can be differentiated by their point of origin (transmission level, distribution level, or between transmission and distribution level). Additionally, one can distinguish congestions between bidding zones and congestions within a bidding zone. The focus of this paper is on the latter one. Since it lays in the nature of a local congestion problem, the geographical information of the offered capacities are of high relevance. $\mathrm{CM}$ includes several measures such as redispatching of conventional power plants, load management and curtailment of renewable energy sources. In the past, a cost-based redispatch approach was the basis for the remuneration of redispatched units. However, a recent court decision [6] clarified that i.a. opportunity costs need to be remunerated as well. Another development is a recent legislative proposal by the European Commission [1] to introduce redispatch markets wherever possible. For the time being a German TSO can request every power plant ( $\geq 10 \mathrm{MW}$ ) to in- or decrease their energy generation compared to their schedule. Power plants are obliged (by EnWG $\S 13$ Abs. 1) to follow these instructions. Due to an unprecedented extension of renewable energy sources and not yet sufficient transmission capacity of the grid, the amount of redispatch strongly grew in the recent years from 2 TWh in 2013 to 16 TWh in 2015 [2] and further increase is expected.

\section{B. Comparison of the market segments}

The timelines of the respective market segments are depicted in Figure 1. The discussed short-term market segments have overlapping time frames, especially in the (so far hypothetical) case of an existing balancing energy and redispatch market.

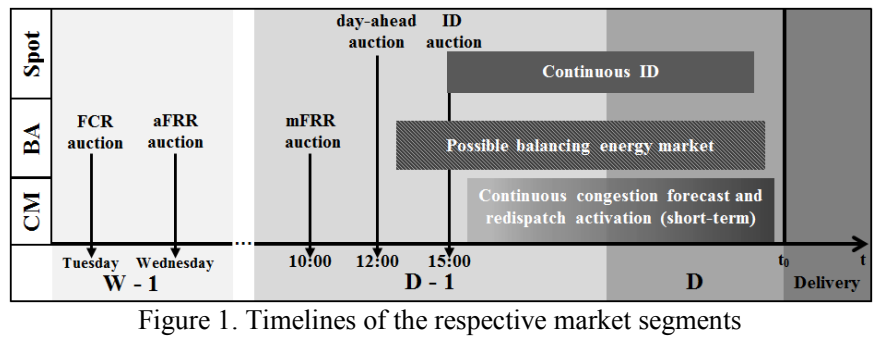

\section{OPTIONS OF INTEGRATING MARKET SEGMENTS}

\section{A. Listing of the options of MSI}

Figure 2 shows the possible four combinations to integrate the three short-term market segments ID, BA and CM.

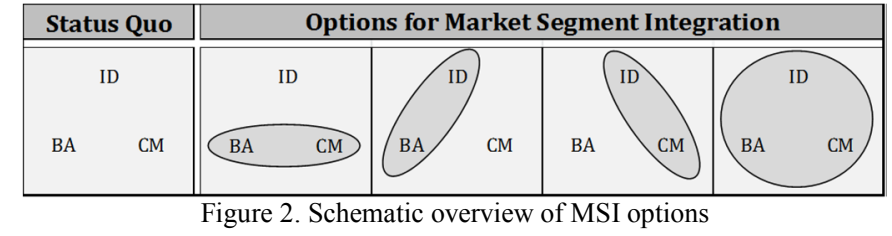

When we talk about integration we do not necessarily mean the complete integration leading to a unified market for the integrated market segments, but also lower degrees of integration. This includes the interaction between and the combination of market segments. ${ }^{2}$ One example for integrating BA and ID is to allow the TSO to procure energy for system imbalance reasons on the ID. We consider the integration of all three market segments is the most visionary MSI option. Due to pragmatic reasons we will particularly focus on the options "integration of BA and CM" as well as "integration of ID and CM" in subchapter B.

\section{B. Description of MSI options}

Every option of MSI represents a design of a highly complex and multifaceted market. Therefore the following descriptions do not aim to finally and completely describe the market design. We will rather determine the basic structure and the fundamental design features.

\section{a) Integration of $B A$ and $C M$}

An example for the integration of the market segments BA and $\mathrm{CM}$ is given by the market design in several countries

\footnotetext{
2 To illustrate, the possibility to optimize the activation of units within the balancing and congestion segment ("co-optimization") differentiates significantly from integrating the respective segments within one market.
} 
within Europe, e.g. the United Kingdom (UK). In its balancing mechanism a co-optimization between BA and CM ensures an efficient use of capacities at all times [7]. Another example is given by the TSO Swissgrid Ltd., who suggested to integrate current products of $\mathrm{mFRR}$ and $\mathrm{CM}$ into one energy product procured by the TSO [8].

For the integration of BA and CM in Germany the BA could be extended such that the TSO is free to activate the bid either for balancing or for congestion purposes ${ }^{3}$. This option is also mentioned in the Electricity Balancing Guideline (EBGL) after which each TSO shall have the right to also activate balancing energy bids for purposes other than balancing, i.e. for ensuring operational security [9]. As both BA and CM, in the current framework, work in a 15 minute pattern and on a scheduled basis, it is appropriate to also orient the integrated design at quarter-hourly products. Therefore, it seems reasonable to consider only the mFRR product for this option of MSI. The inclusion of an aFRR product is also conceivable but adds complexity and is therefore not considered in this paper. Due to CM, every bid needs to entail local information (e.g. grid connection point). Furthermore, the gate opening time (GOT) and GCT for the common market are important design features. In the UK, the TSO receives the first schedule and prices for ramping up or down at 11 a.m. the day before delivery; this schedule can be changed until the GCT of the ID. One option is that the GCT is shortly after or simultaneously with the GCT of the ID. The settlement for respectively up and down regulation can be - in accordance with the EU balancing target model - at a uniform price (marginal pricing) [9].

Currently, the legal framework prohibits the interaction of $\mathrm{BA}$ and $\mathrm{CM}$ in Germany. This is due to the resolution the German regulator Bundesnetzagentur (BNetzA) passed in 2012 that prescribes separation of balancing and congestion measures. According to [10] it is not proportionate to use the redispatch mechanism for balancing the system. Furthermore, the use of reserved balancing capacity for grid issues is prohibited as thereby remaining balancing resources are reduced. In the case of a significant imbalance this could lead to insufficient availability of balancing power [10]. Hence, in an integrated BA and $\mathrm{CM}$ market it needs to be ensured that the dimensioning of reserves takes into account both balancing and congestion needs ${ }^{4}$. However, it needs to be analyzed if a resulting increase in procured capacity in the balancing mechanism is efficient from an economic point of view. The above mentioned balancing energy market, though, is a promising option to introduce a possibility to use these bids for both discussed operational purposes.

\section{b) Integration of ID and CM}

The idea of integrating the ID with the CM in Germany refers to the continuous ID trade - not the auction. This MSI option includes a process whereby the TSO and the Distribution System Operator (DSO) can procure energy for CM purposes on a platform that also performs ID trading of

\footnotetext{
${ }^{3}$ Also other publications discuss this MSI option such as [13].

4 The TSO currently analyses to switch reserve dimensioning from a quarterly process to a process on a daily basis which can be a relevant aspect within this discussion.
}

market parties. Both bids for ID and for CM services could have equivalent product specifications such as a quarterhourly product. Moreover, products require an indication of their physical location of delivery (e.g. grid connection point). A "buy-order" on the ID would constitute a relative energy withdrawal at a specific location. A "sell-order" would subsequently lead to a local energy injection. The system operator would use buy-orders within congested areas, and sell-orders outside congested areas. Such integration has various aspects to be decided on: Within the scope of this paper the ID is not expected to be the exclusive source for CM services but a supplement to other (market-based or nonmarket based) TSO/DSO redispatch processes. The indication of delivery location can be voluntary or obligatory, noting that obligatory indication of location seems somewhat contradictory to zonal electricity market designs. Furthermore, it needs to be defined whether the system operator acts as an active participant by placing orders or a reactive participant only accepting existing bids.

An early GOT gives the TSO the possibility to react on predicted congestions at an early stage. The GCT of this MSI could be - as in the existing continuous ID trade - close to delivery.

\section{DEVELOPMENT OF A CRITERIA-CATALOGUE FOR AN EVALUATION OF THE MARKET CONCEPTS}

\section{A. Definition of criteria}

For an evaluation of any market design model, a list of objective criteria should be the basis to assess both the possible positive and negative consequences. We therefore present the following criteria including a short description to evaluate the two selected MSI options. Although this list of criteria is not necessarily conclusive, we believe that these criteria are crucial when assessing market design options.

\section{a) Market Liquidity}

Market liquidity serves as a quality indicator for a market. Sufficient liquidity enables a functional interaction between supply and demand to find its equilibrium on a settled volume against a certain price. Sufficient liquidity also increases the price deviation resilience if certain bids would be kept out of the market, with a relative small price difference for the settled volume. Due to the multidimensionality of liquidity there is no unequivocal measurand. The different measurands can be categorized into four categories: (1) The transaction cost measures (e.g. the Bid-ask spread), (2) the volume-based measures (e.g. trading volume), (3) the price-based measures and (4) the market impact measures [11].

\section{b) Influence on price formation}

Electricity markets - like every other - consist of market participants that aim to maximize their profit. This understanding explains the central importance of price incentives and revenue possibilities for market participants. Any influences on the price formation process by a MSI should be carefully evaluated. Therefore, possible effects on 
spot, balancing and imbalance prices need to be analyzed. Particular attention is given to possible influences on the imbalance price (IP). The IP [€/ MWh] "means the price, be it positive, zero or negative, in each imbalance settlement period for an imbalance in each direction" [9] It serves as the central incentive for a Balancing Responsible Party (BRP) to be balanced over the ISP and therefore possible influences need to be carefully evaluated.

\section{c) Effect on market participants}

If markets have overlapping time periods, similar products and a similar pool of suppliers, these market participants have a decision problem on which market to offer their flexibility, which is especially a problem for smaller participants. MSI can possibly reduce the decision making problem of market participants and thus improve overall efficiency. Furthermore, adding locational information to bids has various impacts on the market participants. We consider the influence on the revenue structure of market participants and possible distributional effects.

\section{d) Consistency of responsibilities}

In unbundled power systems the market design requires a set of roles that fulfil certain tasks and that have clear responsibilities defined (e.g. TSO, DSO, and BRP). The tasks of the TSO constitute in the BA, where the TSO procures services to balance the power of its control area. A criterion to be evaluated when integrating market segments is the impact on responsibilities: Is the integration leading to an overlap or a gap of responsibilities? Can operator tasks be ensured on integrated markets where the operator is no longer the single-buyer?

\section{e) Market power}

Market power is the ability of single or a small group of market participants to significantly influence the market price. A central measurand for the market power is the market concentration which indicates to which degree only a few market participants control the market. In any integration option which incorporates $\mathrm{CM}$, this issue needs to be discussed. Due to the local character, individual market participants may influence the price formation process. Therefore, it is important to investigate the market power potential and to ensure the existence of appropriate countermeasures. Possible counter-measures are to enlarge the possible pool of providers by e.g. granting access also for small participants or strengthening the market surveillance authority.

\section{f) Supporting system operater's possibilities to (re)act}

It is the responsibility of the TSO to maintain the system security by notably balancing the system and solving congestions. In order to achieve this, sufficient possibilities need to be given to the TSO. This criterion examines whether and to what extent the possibilities of the TSO to procure ancillary services are improved by the respective MSI.

\section{g) Complexity of implementation}

This criterion is dealing with (i) the feasibility of the technical realization and (ii) implementation costs which should not exceed the benefits resulting by a certain MSI option and (iii) the participants that would be directly affected by a conversion (e.g. TSO, generators and power exchanges).

\section{h) Compatibility with the existing regulation}

A possible reorganization of the market design requires an assessment of the compatibility with the regulatory framework and legal basis (e.g. network codes). The current framework for instance foresees a zonal market structure. Therefore it needs to be analyzed if and to what extent MSI could influence or potentially undermine the functionality of the European internal market.

\section{EVALUATION OF THE OPTIONS OF MSI BY MEANS OF SELECTED CRITERIA}

In this section the integration of BA and $\mathrm{CM}$ as well as the integration of ID and CM are evaluated with a subset of the above mentioned criteria. It is not intended as a comprehensive assessment, but rather as an explanatory exercise to show evaluation approaches as well as remaining questions for future research. Therefore, we limit our evaluation to the criteria market liquidity, influence on price formation and effect on market participants.

\section{A. Integration of $B A$ and $C M$}

\section{a) Market Liquidity}

As explained earlier, in the current system the TSO cannot use balancing capacities for solving congestions and vice versa. To be specific, an amount of around $2000 \mathrm{MW}$ is reserved for positive $\mathrm{mFRR}$ and is - under normal conditions - not available for solving congestions. We state that the integration of these segments reduces a liquidity split, which is particularly valid if a balancing energy market and a redispatch market coexist. Bids in this MSI option are used for both imbalance and grid issues. In certain cases, an activated bid could even contribute to balancing the system while solving congestions simultaneously. Even though the possibility of optimization between both is limited, this would improve overall efficiency. Although a quantification of this effect for Germany is still pending, research provides evidence for this argumentation [8]. Furthermore, we argue that an integrated market potentially reduces transaction costs and eliminates arbitrage possibilities if bids from one market are used for several purposes instead of having two parallel segments. Currently, there is a significant overlap of suppliers between both segments also because these segments have similar characteristics.

\section{b) Influence on price formation}

The following analysis shows how price formation is potentially influenced. If the BA and CM are combined there is no direct link to a change of spot prices. However, 
opportunity considerations and a changed revenue expectation could influence prices on the ID.

Regarding balancing and imbalance prices, the EBGL specifies a certain restriction: In case balancing energy bids are activated for purposes other than balancing, the price of these activated balancing energy bids shall not determine the IP [9].

To understand the interdependencies, the calculation of the IP shall briefly be explained. For every quarter hour the cost for the aFRR and the mFRR activation are summed up. This amount is divided by the resulting energy imbalance of the respective ISP.

We use the following simplified example in Figure 3 showing a possible distribution of an integrated market merit order. Bids are either used for congestion (grey) or for balancing (white) purposes.

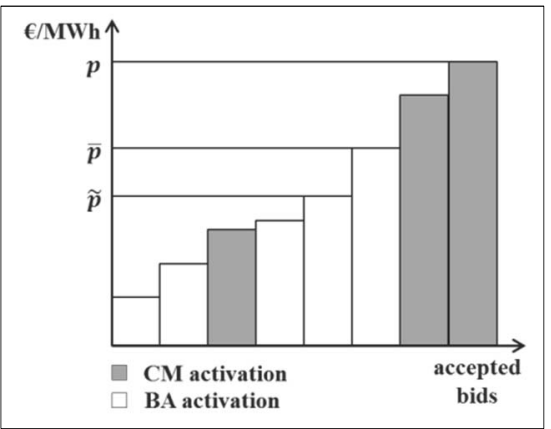

Figure 3: Common merit-order list for both $\mathrm{BA}$ and $\mathrm{CM}$ activation

The marginal price for all activations is $p$. However, as "bids activated for internal congestion management shall not set the marginal price of balancing energy" [9], a different approach is needed. In our example, the highest bid activated for balancing purposes is $\bar{p}$. Still, this marginal price is influenced by the third bid activated to solve a congestion, because otherwise - without an integrated solution - the marginal price for balancing energy would be lower. In both cases the IP is influenced because balancing energy costs are higher compared to the status-quo. One solution could be to calculate a theoretical marginal price $\tilde{p}$ as if there was no bid used for CM. ${ }^{5}$ Such an approach could mitigate undesired influences on the IP.

\section{c) Effect on market participants}

This MSI can have numerous effects on market participants whereby we will focus upon a selection.

In case of an existing separate BA and CM, the MSI would reduce the decision making problem of the market participants if both segments are entirely unified on one platform.

In this MSI - in contrast to the current situation - marginal pricing is assumed, which has an impact on the revenue structure of the market participants. Moreover, locational value is given to a market participant's asset and can lead to

\footnotetext{
${ }^{5}$ Besides this approach, one can argue that as soon as congestion appears, there is not only one marginal price and hence, different marginal values of balancing energy on both sides of the congestion exist.
}

an increase in the producer surplus. ${ }^{6}$ Corresponding increasing cost can arise for the TSO. The cost that occurs for $\mathrm{CM}$ measures are - equivalent to the current situation socialized via grid tariffs.

\section{B. Integration of ID and CM}

\section{a) Market Liquidity}

If the TSO participates passively on the ID and only accepts existing orders we assume that market participants on the ID have priority access to bids and offers (i.e. market orders match first during continuous trade). The TSO uses remaining (i.e. non-matched) orders to not remove liquidity from market participants. Nevertheless, it is possible that the TSO activates bids and offers which could have been activated later in the timescale of the continuous ID.

Looking at the trading volume we can state that, in addition to normal trading, also bids for CM measures are traded on the ID platform. In the case of 2015 the trading volume on the ID in the German-Austrian bidding zone was $38 \mathrm{TWh}$. As a comparison: $8 \mathrm{TWh}$ for each, upward and downward regulation was required for $\mathrm{CM}$ in 2015 [2]. At this stage of research we cannot estimate which share of the $\mathrm{CM}$ could be performed on the ID. But we can state that the trading volume on the ID increases with this MSI option. To specify this evaluation and assess how the liquidity changes for the particular market participants, first the market design needs to be defined in more detail.

\section{b) Influence on price formation}

In this MSI option the TSO is trading on the ID platform, where prices of orders can be accepted that would not have been accepted without the participation of the TSO. Hence, the TSO has an influence on prices on the ID. If the TSO is actively trading on the ID, the influence on prices can be considered to be higher than with a passive participation of the TSO. However, we state that even without an explicit integration of market segments there are interdependencies between market segments due to opportunity considerations.

The IP is not directly influenced by this MSI. However, since 2012 the IP is coupled with the ID price to prevent profit opportunities from an imbalanced BRP [12]. As the ID price is influenced, also the IP is indirectly influenced as often as this coupling takes place. In 2015 a coupling with the ID took place $7 \%$ of the time. We expect that this MSI has a direct influence on the ID prices and an indirect influence on the IP.

\section{c) Effect on market participants}

This MSI option can have numerous effects on market participants whereby we will focus upon a selection.

In case of an existing separate ID and CM market, the MSI would reduce the decision making problem of the market participants. This statement is not valid if the MSI poses an additional bid option for the market segments.

\footnotetext{
${ }^{6}$ Please note that the potential locational value results from a market-based redispatch regime and not from the MSI itself.
} 
As the obligatory locational information needs to be given in this MSI, the market participants have to trade for their specific units and cannot optimize their portfolio to the current extent.

Furthermore, we expect a change of the revenue structure. By giving the TSO the possibility to solve redispatch issues via the ID, locational value is given to a market participants asset and can lead to an increase in the producer surplus. Corresponding increasing cost can arise for the TSO. The cost that occurs for $\mathrm{CM}$ measures are - equivalent to the current situation - socialized via grid tariffs.

We therefore expect various effects on the different market participants.

\section{CONCLUSION}

This paper discusses options for the future short-term market design. In particular, we develop a conceptual basis for a possible integration of currently separated short-term market segments. Market segment integration (MSI) is defined as the interaction between and possible combination of market segments. Thereby an integration of market segments does not necessarily lead to a unified market for the integrated market segments but in our understanding also includes lower degrees of integration that allows for certain interactions. For the integration option BA and $\mathrm{CM}$ as well as ID and CM, we determine the basic design features such as product specifications, settlement structure or temporal aspects. A profound list of central criteria enables the evaluation of MSI. We evaluate a selection of criteria for the illustrated MSI options and present both positive (e.g. an increase of liquidity) and negative (e.g. possible distortions on price formation) effects. Even without any integration, there are interdependencies between market segments if market parties need to choose where to trade flexibility and arbitrage possibilities exist. MSI addresses these interactions and tries to develop potential solutions for emerging problems.

This paper opens up a wide range of possibilities for further research. The design for the MSI options can be specified in more detail for the options to integrate the ID and the $\mathrm{CM}$ as well as the option to integrate the BA and CM. Also the concrete design of the two MSI options that have not been addressed in this paper can be concretized and analyzed with the provided criteria catalogue. Along with ongoing concretization of the MSI design, the evaluation can be expanded to more quantitative measures.

\section{DISCLAIMER}

Opinions expressed in this paper are those of the authors and do not necessarily reflect views of the companies/the institute.

\section{REFERENCES}

[1] European Commission, "https://ec.europa.eu," 3011 2016. [Online]. Available: https://ec.europa.eu/energy/en/news/commissionproposes-new-rules-consumer-centred-clean-energy-transition. [Accessed 2702 2017].

[2] Bundesnetzagentur, "www.bundesnetzagentur.de," [Online]. Available:

https://www.bundesnetzagentur.de/DE/Allgemeines/Presse/Mediath ek/Berichte/berichte-node.html. [Accessed 2702 2017].

[3] EPEX SPOT, "www.epexspot.com," 1101 2017. [Online]. Available: https://www.epexspot.com/en/pressmedia/press/details/press/EPEX SPOT_Intraday markets_reach_all -time_high_in_2016. [Accessed 2702 2017].

[4] Regelleistung.net, "www.regelleistung.net," 2702 2014. [Online]. Available: https://www.regelleistung.net/ext/static/marketinformation?lang=en. [Accessed 2702 2017].

[5] 50Hertz Transmission GmbH; Amprion GmbH; TenneT TSO GmbH; Transnet BW GmbH, "http://www.tennet.eu/de," 1407 2016. [Online]. Available:

http://www.tennet.eu/fileadmin/user_upload/Company/Publications/ Position_Papers/German $/ \% \mathrm{C} 3 \% 9 \mathrm{CNB}$ _Stellungnahme_Wei $\% \mathrm{C} 3 \%$ 9Fbuch_Ein_Strommarkt_f $\%$ C3\%BCr_die_Energiewende_20.08.20 1....pdf. [Accessed 2702 2017].

[6] OLG Düsseldorf, VI-3 Kart 332/12, Düsseldorf, 2015.

[7] National Grid, "www2.nationalgrid.com," [Online]. Available: http://www2.nationalgrid.com/WorkArea/DownloadAsset.aspx?id= 8589934825. [Accessed 2702 2017].

[8] A. Troupakis, M. Vrakopoulou, F. Abbaspourtorbati, G. Andersson and M. Zima, Market clearing framework for an integrated market for manually activated control reserves and redispatch in Switzerland, 2014.

[9] ENTSO-E, "ENTSO-E Network Code on Electricity Balancing," 25 01 2017. [Online]. Available: https://www.entsoe.eu/majorprojects/network-code-development/electricitybalancing/Pages/default.aspx. [Accessed 2702 2017].

[10] Bundesnetzagentur, BK6, Beschluss - BK6-11-098, Bonn, 2012.

[11] A. Sarr and T. Lybek, Measuring Liquidity in Financial Markets, International Monetary Fund, 2002.

[12] Regelleistung.net, "www.regelleistung.net," [Online]. Available: https://www.regelleistung.net/ext/static/rebap. [Accessed 2702 2017].

[13] Consentec, "Methodische Fragen bei der Bewirtschaftung innerdeutscher Engpässe im Übertragungsnetz (Energie)," Bonn, 2008 . 